\title{
Assessing Fear of Recurrence of Esophageal Cancer in Patients Treated with Esophagectomy
}

Johnathan Brown, MD; Annemarie Opipari, MD; Nathan Rietberg, MD; Daniel Klarr, MD; Michael Hipp, BS; Anita Pandit, MS; Lili Zhao, PhD; Susan G. Urba, MD; Shari Barnett, BS; Judy Miller; Philip W. Carrott, MD; William Lynch, MD; Rishindra M. Reddy, MD; Jules Lin, MD; Mark B. Orringer, MD; Andrew C. Chang, MD

\section{ABSTRACT}

\section{Background}

Fear of cancer recurrence is prevalent among esophageal cancer patients. Little to no treatment is available for this problem. We evaluated the overall quality of life (QoL) following esophagectomy, symptom-specific quality of life, and symptoms related to fear of recurrence (FoR). Our analysis intended to determine the relationships between QoL, symptom-specific QoL, and symptoms related to FoR in order to examine how to potentially address the various elements affecting QoL and FoR.

\section{Methods}

FoR and QoL were assessed prospectively with the "Fear of Recurrence" survey as well as the EORTC QLQ-C30 and esophageal cancer module (OES18) QoL surveys, administered preoperatively, immediately postoperatively, and at 6 and 12 months after esophagectomy. Demographics, clinical parameters, and outcomes were extracted from a prospective institutional database. From February 2010 to April 2015, 222 patients enrolled in this study and underwent esophagectomy. A linear mixed effects model assessed correlation between measures of patient anxiety and quality of life. A random effect was included in the model to account for a correlation between measurements within the same patient. The same model compared patient anxiety and quality of life at the measured time points. A Cox proportional hazards model was used to estimate the effect parameter of physical symptoms on the fear of recurrence while adjusting for the decreasing response rate.

\section{Results}

With median follow-up of 10 months (range 0-63), 160, 81, and 40 subjects completed the postoperative surveys immediately 
after surgery and at 6 months and 12 months, respectively. Patient anxiety, as assessed by FoR scores, decreased initially $(p<0.001)$ in the immediate postoperative and 6-month periods compared to the baseline preoperative score but returned to baseline within 1 year. At the first postoperative assessment, patient anxiety was associated with symptoms including fatigue, pain, dyspnea, loss of appetite, and postoperative pain $(p<0.0001)$. Fear of recurrence scores were associated negatively $(p<$ 0.0001 ) with functioning status across all domains assessed by the QLQ-C30.

\section{Conclusions}

Patients' fear of cancer recurrence has a pattern of decreasing from the preoperative baseline

\section{Introduction}

E sophageal cancer is associated with high morbidity and mortality. Worldwide, esophageal cancer is the eighth most common malignancy. ${ }^{1}$ While esophageal resection with or without chemotherapy or chemoradiotherapy remains a mainstay in the treatment of esophageal cancer, this operation has a high rate of postoperative complications ${ }^{1}$ and carries considerable long-term sequelae. For patients who undergo operative treatment through surgical resection, the 5 -year survival rate is only $31 \%$. The rate of recurrence following treatment is also high. A retrospective review has shown as many as 38\% of patients developed a recurrence of their cancer over a period of 16 years following operative treatment. ${ }^{1}$ Clinical symptoms were the first indication of recurrence in half of the cases. ${ }^{1}$ Additionally, perioperative complications can impact not only long-term survival but also quality of life for the patient. It has been shown that social function is negatively affected among patients who undergo esophagectom. ${ }^{3,4}$ Several investigators have established an interaction between operative outcomes and functional decline following esophagectomy, but the relationship between patient anxiety, over a period of 6 months postoperation but returns to baseline levels 1 year following the esophagectomy. Additionally, the symptoms of fatigue, pain, dyspnea, loss of appetite, and postoperative pain are shown to increase patient anxiety following an esophagectomy. The increase in FoR is important because higher levels of FoR are associated with decreased functional status across all domains. Addressing QoL, symptomspecific QoL, and FoR through direct and indirect interventions may help improve QoL and lessen FoR. Further studies should examine which interventions are effective and more deeply examine the complex relationship between the variables to determine causality in QoL and FoR scores.

health-related quality of life (QoL), and outcomes remain unclear. Fear of cancer recurrence has been a focus of cancer survivorship studies but has not yet been evaluated in the population of patients who are postoperative from esophagectomy for treatment of esophageal cancer. Furthermore, prior studies have not examined which physical symptoms have the greatest impact on quality of life and fear of recurrence (FoR).

In our study, we sought to identify physical symptoms associated with decreased QoL and increased FoR. We believe that a subset of a few physical symptoms account for the majority of the decrease in QoL and increase in FoR. Given that most validated QoL and FoR surveys use a litany of symptoms in their calculations, a subset of these symptoms is likely the symptoms of interest for this study. Utilizing a validated FoR survey ${ }^{5}$ along with well-established QoL surveys (EORTCQLQ30 and OES18) for esophageal cancer, we studied a cohort of esophageal cancer patients and evaluated their overall health-related QoL, symptom-specific QoL, and FoR in order to better understand these factors in the setting of a high morbidity treatment. Ideally, by finding which physical symptoms most affect QoL and FoR, we 
believe that future therapies can be targeted to treat the symptoms, which could be used in future studies to determine causation versus correlation between the symptoms, FoR, and QoL.

\section{Methods \\ Participants}

This study was first approved by the University of Michigan Institutional Review Board. Fear of recurrence (FoR) and patient quality of life (QoL) were assessed from prospectively obtained surveys from esophageal cancer patients undergoing esophagectomy. The patients were recruited by thoracic surgeons at Michigan Medicine. All patients at Michigan Medicine undergoing an esophagectomy for esophageal cancer were included. Exclusion criteria were patients not undergoing esophagectomy at Michigan Medicine or those undergoing esophagectomy for some other reason. There were no incentives for participation. Three types of esophagectomies were included in the study, and the pathological stage of cancer ranged from 0 to III. The difference in surgical techniques, pathologic stage, and other demographics were not evaluated in this study. Demographics, clinical parameters, and outcomes were extracted from our institutional prospectively maintained database.

\section{Measures}

The "Fear of Recurrence" survey as well as the European Organisation for Research and Treatment of Cancer (EORTC) QLQ-C30 (version 3.0)6,7 and esophageal cancer module (OES18) ${ }^{8}$ QoL surveys were administered at specified time points to 222 patients. The Fear of Recurrence survey was originally developed for post-mastectomy patients, but the questions had been modified for patients post-esophagectomy. The EORTC QLQC30 is a questionnaire of both multi-item and single-item measures designed to assess QoL of cancer patients in international clinical trials. The OES18 is a complementary questionnaire to the QLQ-C30 and uses a Likert scale to measure QoL of esophageal cancer patients in clinical trials. The QoL surveys asses global QoL, physical functioning, role functioning, emotional functioning, cognitive functioning, and social functioning. The surveys also assess the severity of the following symptoms: fatigue, nausea and vomiting, pain, dyspnea, insomnia, loss of appetite, constipation, diarrhea, and financial difficulties.

\section{Procedures}

The specified time points were preoperation, immediately postoperation, 6 months postoperation, and 12 months postoperation. The Fear of Recurrence survey measures patient anxiety through 20 questions on a Likert type scale ranging from 1 = almost never to 4 = almost always. The total survey scores were measured rather than analyzing individual question scores. Higher scores indicate greater anxiety. Survey assessments were preferably conducted face-to-face in the clinic setting when possible. If a participant missed scheduled postoperative follow-up at our institution and the preferred face-to-face interview was not possible, the survey assessments were mailed to the patient's residence with a self-addressed, stamped return envelope. To maximize recovery of longterm survey assessments, follow-up phone calls were placed to the participant's residence if return surveys were not received within 1 month from the time of mailing. The response rate at each point in the study was calculated by dividing the number of responses by total number of living participants. In addition to FoR, QoL, and symptoms were recorded through the surveys at the 4 time points.

\section{Statistical Analyses}

Correlation between measures of patient anxiety and quality of life were assessed using a linear mixed effects model, and a random effect was included in the model to consider the correlation between measurements within the same patient. The same model was used to compare the difference between mean scores of patient anxiety and quality of life at the measured time points. To 
account for the decreasing response rate and low survival rate, a Cox proportional hazards model was used. Cox proportional hazards model was used to estimate the effect parameter of physical symptoms on QoL and FoR by accounting for the diminishing response rate and low survival rate following esophagectomy. The effect parameter estimate could then be used to evaluate which physical symptoms were correlated with the greatest increase in FoR. Statistical significance was determined if $p<0.05$. All analyses were conducted using SAS (version 9.4, SAS Institute, Cary, NC).

\section{Results}

From February 2010 to April 2015, 222 subjects underwent esophagectomy at a single tertiary referral center and completed surveys assessing FoR and health-related QoL. The transhiatal surgical approach was used for most patients (Table 1). Most of the participants in the study were Caucasian males with a Zubrod score (measure of performance status in cancer patients) of 0 to 1 (Table 2). Survey response rates decreased during the study of this period to $22.6 \%$ among enrolled survivors at 1 year following operation (Table 3-online only). Most notably, 45 of the original 222 patients were deceased 1 year following the operation, which was $20.2 \%$ of the original patient sample size.

Sample questions from the Fear of Recurrence survey are shown in Table 4-online only. ${ }^{5}$ The FoR trended down immediately postoperation compared to preoperation scores $(p=0.0002)$. Anxiety, as denoted by the Fear of Recurrence survey, increased at 6 months and 12 months following esophagectomy compared to the lowest score immediately postoperation (Figure 1). The Fear of Recurrence score at the immediate postoperation and 6-month time points showed statistically significant decreases ( $p=0.0059)$ compared to the preoperation score (Table 5-online only). At 12 months following operation, patient anxiety had returned to preoperation levels $(p=0.2241)$.

The patient-reported symptoms measured by the QLQ-C30 and OES-18 surveys showed significantly decreased physical, social and role functioning, and global health status at the first postoperative time point ( $p<0.0001$ ) compared to the preoperative assessment with recovery of functional status close to baseline function by the 6-month postoperative time point (Figure 2). Measures of cognitive and emotional functioning did not change significantly during the first

\section{TABLE 1. Operative Characteristics}

\section{Surgical Approach}

Transhiatal

Minimally Invasive Esophagectomy

McKeown

\section{Stage, Pathologic (yp) $($ Total $=222)$}

I

II

III

IV

Unknown

II

$0 \quad 52(23.4 \%)$

\section{N (\%)}

$191(86.0 \%)$

$21(9.5 \%)$

$10(4.5 \%)$ $59(26.6 \%)$

$56(25.2 \%)$

$54(24.3 \%)$

$0(0.0 \%)$

$1(0.5 \%)$

$50 \cdot$ MICHIGAN JOURNAL OF MEDICINE 2018 
postoperative year (Figure 2). The QLQ-C30 and OES-18 surveys revealed several variables that correlated with the fear of recurrence of esophageal cancer. Most of the patient-reported functioning scores such as global health status, cognition, and social functioning were inversely related to the FoR ( $p<0.0001$ ) (Table 6). Symptom scores, particularly nausea or vomiting, pain, and fatigue correlated directly with self-reported FoR $(p<0.0001)$ (Table 5-online only).

TABLE 2. Subject Characteristics

\begin{tabular}{ll}
\hline Characteristic & N (\%) \\
Age & \\
\hline Median & 64 \\
\hline Range & $33-90$ \\
\hline Sex & \\
\hline Female & $26(11.7 \%)$ \\
\hline Male & $196(88.3 \%)$ \\
\hline Race (Total = 222) & \\
\hline Caucasian & $216(97.7 \%)$ \\
\hline Black/African American & $4(1.8 \%)$ \\
\hline Asian & $1(0.5 \%)$ \\
\hline No Response Provided & $1(0.5 \%)$ \\
\hline American Society of Anesthesiologists (ASA) Score & \\
\hline I & $2(0.9 \%)$ \\
\hline II & $76(34.2 \%)$ \\
\hline III & $138(62.2 \%)$ \\
\hline IV & $6(2.7 \%)$ \\
\hline Zubrod Score & $6(2.7 \%)$ \\
\hline 0 or 1 & \\
\hline 2 or 3 & $216(97.3 \%)$ \\
\hline
\end{tabular}

TABLE 3. ONLINE ONLY-Survey Completion

TABLE 4. ONLINE ONLY_Sample Questions from the Fear of Recurrence Survey used for Esophagectomy Patients

TABLE 5. ONLINE ONLY_Comparison of Fear of Recurrence Scores between the Various Time Points in the Study 


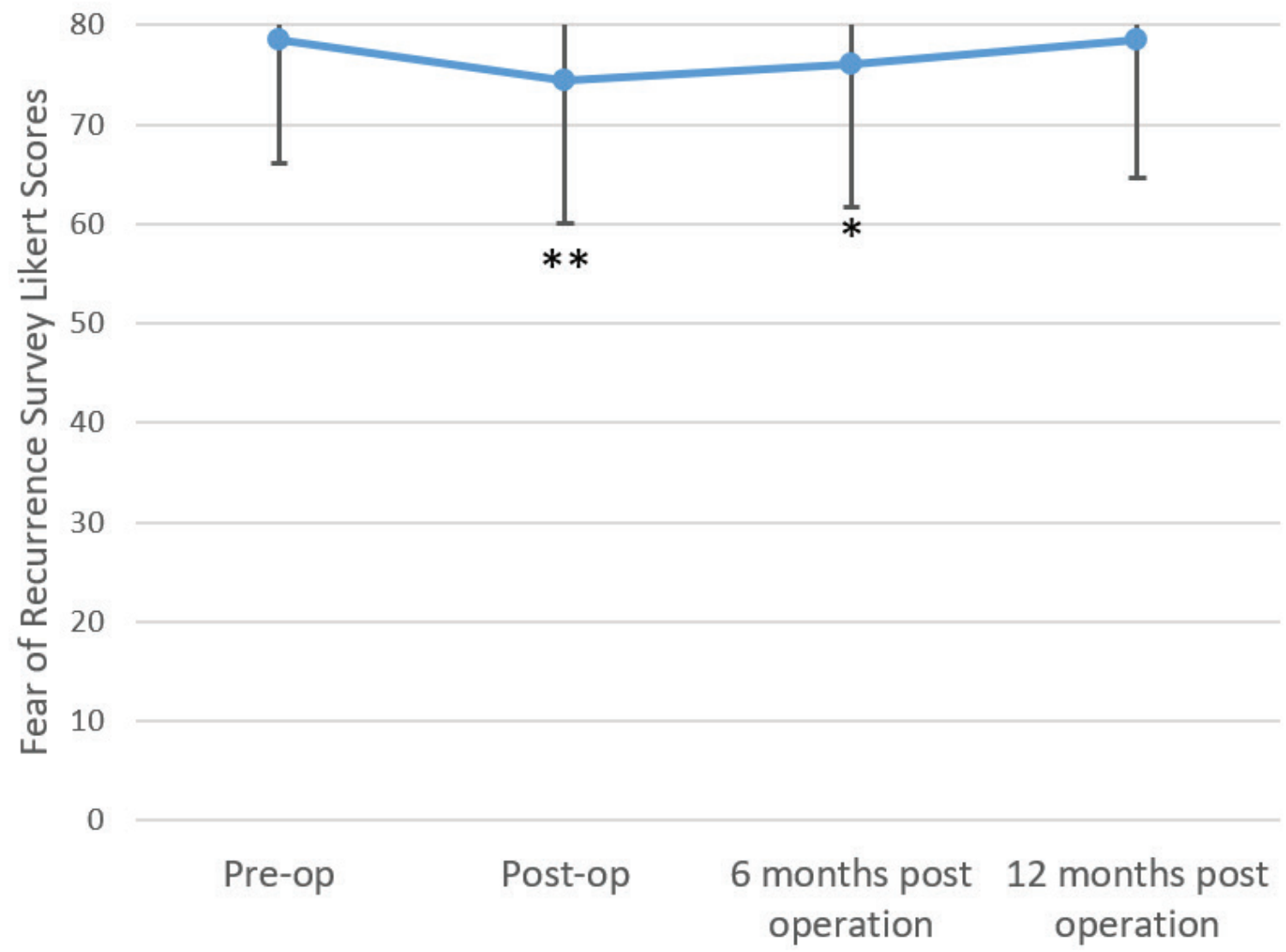

FIGURE 1. Fear of Recurrence Score Trend from Preoperation to 12 Months after the Operation

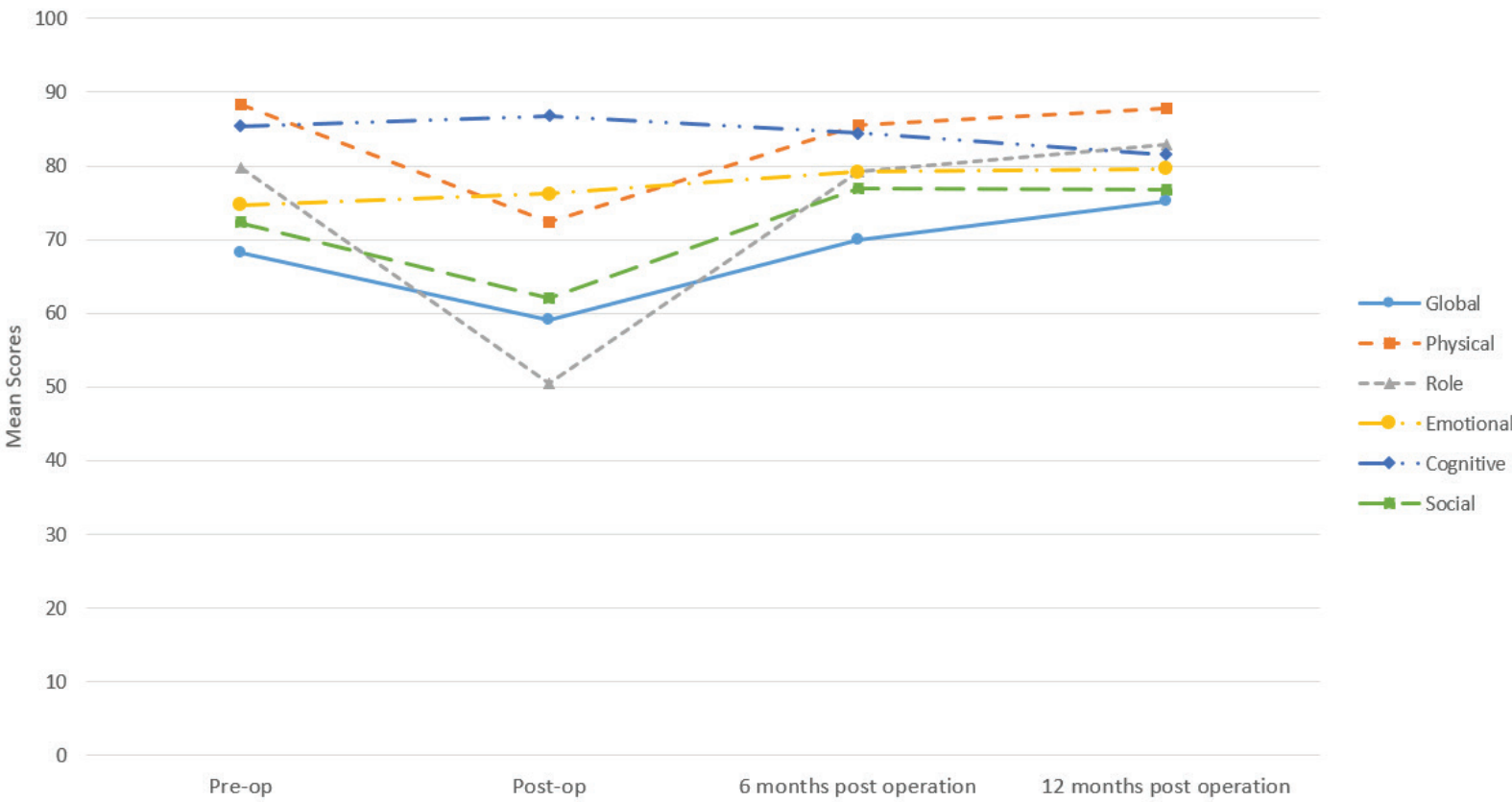

FIGURE 2. Perioperative and Postoperative Function Scores (QLQ-C30) 
TABLE 6. Symptoms Correlating with Fear of Recurrence

\begin{tabular}{lll}
\hline Effect & \multicolumn{1}{l}{$\begin{array}{l}\text { Effect Parameter } \\
\text { Estimate on FoR }\end{array}$} & p-value \\
\hline Nausea and Vomiting & 0.1382 & $<.0001$ \\
\hline Pain (OES18) & 0.1361 & $<.0001$ \\
\hline Fatigue & 0.1231 & $<.0001$ \\
\hline Pain & 0.1176 & $<.0001$ \\
\hline Eating & 0.1097 & $<.0001$ \\
\hline Insomnia & 0.102 & $<.0001$ \\
\hline Constipation & 0.08058 & 0.0007 \\
\hline Appetite Loss & 0.0749 & $<.0001$ \\
\hline Reflux & 0.07062 & 0.0079 \\
\hline Financial Difficulty & 0.0688 & 0.0012 \\
\hline Taste & 0.05891 & 0.0008 \\
\hline Dyspnea & 0.05608 & 0.0199 \\
\hline Dry Mouth & 0.04966 & 0.0138 \\
\hline Role Functioning & -0.0511 & 0.0043 \\
\hline Physical Functioning & -0.1034 & 0.001 \\
\hline Social Functioning & -0.1275 & $<.0001$ \\
\hline Global Health Status & -0.1507 & $<.0001$ \\
\hline Cognitive Functioning & -0.1877 & $<.0001$ \\
\hline Emotional Functioning & -0.3265 & $<.0001$ \\
\hline
\end{tabular}

\section{Discussion}

This study indicates that, following an esophagectomy, patient anxiety, specifically fear of recurrence, initially drops during the postoperation period but begins to increase by 6 months postoperation (Figure 1). The Fear of Recurrence survey measure correlates with severity of nausea and vomiting, fatigue, and pain. While FoR had increased 6 months after operation compared to the immediate postoperative levels, patients' global health, physical functioning, social functioning, and role functioning also returned to baseline within the same 6-month time span. Notably, the Fear of Recurrence survey appeared to suggest greater patient anxiety than that determined by the QLQ-C30 measure of emotional functioning, which remained stable during the measured time points. The return to baseline is consistent with similar studies evaluating QoL among esophagectomy patients.4,9,10 These results indicate that multiple factors are associated with patient anxiety in regards to cancer recurrence.

The findings indicate that the severity of fatigue, nausea and vomiting, and pain several months after surgery may correlate with the level of a patient's fear of recurrent malignancy. Additionally, there is a positive directionality of correlation between the severity of symptoms and FoR despite the absence of proof of causality. Patients who have considerably greater severity of symptoms 6 months and 12 months after esophagectomy were likely to have higher levels of anxiety. This subset of physical symptoms has a major impact on FoR as hypothesized. However, we were unable to determine if these symptoms alone accounted for the majority of the FoR, because there were other significant factors such as insomnia and eating difficulties.

By effectively measuring a patient's FoR through the already measured levels of fatigue, nausea and vomiting, and pain, perhaps providers could limit patient anxiety through better treating patient symptoms. It may be possible that these symptoms heighten patient anxiety, because clinical symptoms can result in the detection of recurrence in nearly half of the cases. ${ }^{1}$ By addressing these symptoms when there are no indications of recurrence during follow-up, physicians may be able to allay this anxiety. This practice has been shown to be effective in other chronic symptoms such as those seen in heart failure. ${ }^{11}$ Further treatment may include supportive care to handle fatigue, nausea and vomiting, and pain or counseling to directly manage anxiety. Future studies could be done to examine the effectiveness of treating these symptoms to lessen FoR and to determine if causality between the symptoms and FoR is present. 
Developing a method to address the symptoms and FoR may lead to future improvements in the overall QoL for patients following esophageal resection. QoL baseline for patients should be defined preoperatively when a patient has been diagnosed with esophageal cancer to best gauge the effectiveness of treatments given. In light of the complex relationship between patientreported symptoms, anxiety, and overall survival, an underlying causation between symptoms and FoR may be possible based on the positive correlation shown in this study. If causation is present, interventions that can both directly and indirectly address the symptoms and anxiety throughout the phases of peri- and postsurgical care may result in meaningful improvement in FoR and overall functional status following morbid operations such as esophagectomy.

There were several limitations of our study, especially related to the study population and attrition rate. One limitation of this study includes its completion at a single institution over a 5-year time span in a relatively homogenous population. The homogenous group makes it difficult to extrapolate the results to other institutions whose populations may greatly vary from the study participants. Additionally, the number of respondents decreased from 222 subjects who completed the preoperative baseline study to 40 subjects who completed the 12-month postoperative survey. The large decrease in subjects cannot be fully accounted for with the limited information obtained through the surveys. A multitude of factors are likely the cause of the decrease. Some of the factors to consider include death of patients related to the high mortality of esophagectomy and esophageal cancer, patient withdrawal from the study, and failure to return surveys. Nonetheless, the high attrition rate weakens the statistical models used to determine the connections between the variables despite the use of Cox proportional hazards model to account for some of the impact. Another minor limitation of the study is the variation in the type of esophagectomy. Although most patients had received transhiatal esophagectomy ( $N=191)$, remaining subjects had undergone either minimally invasive esophagectomy ( $N=21$ ) or three-field esophagectomy $(N=10)$. The type of surgery has potential to be confounding the results slightly. Minimally invasive esophagectomy and three-field esophagectomy only consist of $13.9 \%$ of all data points; therefore, the type of surgery would likely have a limited, small effect if there is one present.

\section{Conclusion}

Following esophagectomy, patients may experience a higher fear of recurrence if their symptoms, especially fatigue, nausea and vomiting, and pain, are not adequately addressed. This study also suggests that anxiety related to fear of cancer recurrence is influenced by multiple factors that are measured using established quality of life assessments. Fatigue, nausea and vomiting, and pain several months after an esophagectomy positively correlate with a fear of recurrence. Symptom management can potentially be used to ameliorate patient anxiety related to the cancer diagnosis. Treating anxiety either directly or indirectly is a common problem throughout healthcare and worthy of study in relation to esophagectomies. For example, other studies examining care following acute trauma such as myocardial infarction and stroke have found that more investigation is needed for providers to recognize and better respond to patient anxiety. ${ }^{12,13}$ Future studies on the appropriate level of postoperative care patients should receive after an esophagectomy to address their fear of recurrence, symptoms, and quality of life would be of benefit.

\section{Acknowledgments}

This work was supported by funding from the John Alexander Distinguished Professorship, Section of Thoracic Surgery, University of Michigan, Ann Arbor, MI. 


\section{References}

1. Lou F, Sima CS, Adusumilli PS, et al. Esophageal cancer recurrence patterns and implications for surveillance.J Thorac Oncol. 2013;8(12):1558-1562. doi:10.1097/01.JTO.0000437420.38972.fb

2. Raymond DP, Seder CW, Wright CD, et. al. Predictors of major morbidity or mortality after resection for esophageal cancer: a Society of Thoracic Surgeons General Thoracic Surgery Database risk adjustment model. Ann Thorac Surg. 2016;102(1):207214. doi:10.1016/j.athoracsur.2016.04.055

3. Malmström M, Klefsgard R, Ivarsson B, Roman $M$, Johansson J. Quality of life measurements as an indicator for timing of support after oesophagectomy for cancer: a prospective study. BMC Health Serv Res. 2015;15:96. doi:10.1186/s12913-015-0747-x

4. Barbour AP, Lagergren P, Hughes R, Alderson D, Barham CP, Blazeby JM. Health-related quality of life among patients with adenocarcinoma of the gastro-oesophageal junction treated by gastrectomy or oesophagectomy. BritJ Surg. 2008;95(1):8084. doi:10.1002/bjs.5912

5. Northouse LL. Mastectomy patients and the fear of cancer recurrence. Cancer Nurs. 1981;4(3):213-220.

6. Aaronson NK, Ahmedzai S, Bergman B, et al. The European Organization for Research and Treatment of Cancer QLQ-C30: a quality-of-life instrument for use in international clinical trials in oncology. J Natl Cancer Inst. 1993;85(5):365-376.

7. Bjordal K, de Graeff A, Fayers PM, et al. A 12 country field study of the EORTC QLQ-C30 (version 3.0) and the head and neck cancer specific module (the
EORTC QLQ-H\&N35) in head and neck patients. Eur J Cancer. 2000;36(14):213-219. doi:10.1016/S0959 -8049(00)00186-6

8. Blazeby JM, Alderson D, Winstone K, et al. Development of an EORTC questionnaire module to be used in quality of life assessment for patients with oesophageal cancer. Eur J Cancer. 1996;32(11):1912-1917. doi:10.1016/0959-8049 (96)00199-2

9. Sanghera SS, Nurkin SJ, Demmy TL. Quality of life after an esophagectomy. Surg Clin North Am. 2012;92(5):1315-1335. doi:10.1016/j.suc.2012.07 .001

10. Trudel JG, Sulman J, Atenafu EG, Kidane B, Darling GE. Longitudinal evaluation of trial outcome index scores in patients with esophageal cancer. Ann Thorac Surg. 2016;102(1):269-275. doi:10.1016/j .athoracsur.2016.01.091

11. Diop MS, Rudolph JL, Zimmerman KM, Richter MA, Skarf LM. Palliative care interventions for patients with heart failure: a systematic review and metaanalysis. J Palliat Med. 2017;20(1):84-92. doi:10 .1089/jpm.2016.0330

12. White $C L$, Barrientos R, Dunn K. Dimensions of uncertainty after stroke: perspectives of the stroke survivor and family caregiver. I Neurosci Nurs. 2014;46(4):233-240. doi:10.1097/JNN .0000000000000066

13. Jones J, Kane P, Polson R, et al. Protocol for a systematic review of screening tools for fear of recurrent illness in common life-threatening diseases. Syst Rev. 2015;4:10. doi:10.1186/2046-4053-4-10 